\title{
Hyperspectral pan-sharpening: a variational convex constrained formulation to impose parallel level lines, solved with ADMM
}

\author{
Alexis Huck, François de Vieilleville, Pierre Weiss and Manuel Grizonnet
}

\begin{abstract}
In this paper, we address the issue of hyperspectral pan-sharpening, which consists in fusing a (low spatial resolution) hyperspectral image $\mathrm{HX}$ and a (high spatial resolution) panchromatic image $P$ to obtain a high spatial resolution hyperspectral image. The problem is addressed under a variational convex constrained formulation. The objective favors high resolution spectral bands with level lines parallel to those of the panchromatic image. This term is balanced with a total variation term as regularizer. Fit-to-P data and fit-to-HX data constraints are effectively considered as mathematical constraints, which depend on the statistics of the data noise measurements. The developed Alternating Direction Method of Multipliers (ADMM) optimization scheme enables us to solve this problem efficiently despite the non differentiabilities and the huge number of unknowns.
\end{abstract}

Index Terms-hyperspectral, fusion, pan-sharpening, ADMM

\section{INTRODUCTION}

High spectral resolution of hyperspectral imaging sensors generally implies concession on spatial resolution due to optics/photonics and cost considerations.

If high spatial resolution panchromatic data are available, hyperspectral pan-sharpening can significantly help to improve the spatial resolution of sensed hyperspectral images.

In the last three decades, pan-sharpening approaches were dedicated to multispectral data. The earliest methods were based on specific spectral-space transforms such as the HueIntensity-Saturation (HIS) transform or the Principal Component Analysis (PCA) Transform. More recently, spatial frequency based approaches such as the High Pass Filter (HPF) method exploiting multiscale spatial analysis [1] provided improved results. The multiscale spatial analysis framework generally offers very time efficient performance but lacks flexibility to consider some prior knowledge about "physics of scene and sensor" (the sensors Modulation Transfer Function (MTF), sensor noise or any prior information). This aspect has been a limitation for application to hyperspectral pansharpening. Thus, recent methods are generally based on variational [2] or bayesian [3] formulations. In particular, in [2], the authors have proposed to consider a term based on the topographic properties of the panchromatic image. This idea stems from [4] where the authors show that most geometrical information of an optical image lies in the set of its gray levelset lines.

A. Huck and F. De Vieilleville are with Magellium, Toulouse, France. P. Weiss is with ITAV-USR3505, université de Toulouse, France,

M. Grizonnet is with CNES, Toulouse, France.
The proposed algorithm includes three novelties: 1 . we propose a constrained convex formulation where the constraints are the fit-to-data terms. This enables to easily tune the related parameters which are the (supposed) known noise variances of the sensors. 2. The proposed minimization algorithm is based on the ADMM. It handles the non differentiabilities, constraints and special structures of the linear transforms in an efficient way. 3. The formulation takes the MTF (Modulation Transfer Function) into account, which helps refining the fit-tohyperspectral-data constraint. This is favorable to high spectral fidelity in pan-sharpened hypersepctral data.

\section{Problem Formulation}

In this paper, we rearrange (hyperspectral) images into vectors in order to allow writing matrix-vector products. Let $x=\left(\begin{array}{c}x_{1} \\ \vdots \\ x_{L}\end{array}\right) \in \mathbb{R}^{L M}$ and $u=\left(\begin{array}{c}u_{1} \\ \vdots \\ u_{L}\end{array}\right) \in \mathbb{R}^{L N}$ denote the low spatial resolution (LR) measured hyperspectral image and the (unknown) high spatial resolution hyperspectral image respectively. The integers $L$ and $M$ represent the number of spectral bands and the number of spectral pixels in the low resolution image, respectively. We let $p \in \mathbb{R}^{N}$ denote the rearranged panchromatic measured image, where $N=q^{2} \times M$ and $q \geq 1$ denotes the resolution factor between the low and high resolution images. The linear projection operator which returns the $l^{t h}$ spectral band is denoted $\pi_{l}$. Then, $x_{l}=\pi_{l} x \in \mathbb{R}^{M}$ and $u_{l}=\pi_{l} u \in \mathbb{R}^{N}$ are the $l^{t h}$ spectral bands of $x$ and $u$ respectively.

A model formulation for any spectral band $l$ of the hyperspectral measurements is given by

$$
x_{l}=\mathbf{D}_{s} \mathbf{H}_{s} u_{l}+n_{x_{l}} .
$$

The linear operator $\mathbf{H}_{s} \in \mathbb{R}^{N \times N}$ respresents the spatial convolution with the spatial Point Spread Function of the hyperspectral sensor. The linear operator $\mathbf{D}_{s} \in \mathbb{R}^{M \times N}$ is a downsampling operator that preserves 1 every $q$ pixels in the horizontal and vertical directions. Some additive sensor noise is considered in the vector $n_{x_{l}}$. We assume that $n_{x_{l}} \sim$ $\mathcal{N}\left(0, \sigma_{x_{l}}^{2}\right)$ where $\sigma_{x_{l}}^{2}$ is the noise variance of the $l^{t h}$ measured hyperspectral band.

A model formulation for the panchromatic image acquisition process is given by

$$
p=\mathbf{G} u+n_{p}
$$


where $\mathbf{G} \in \mathbb{R}^{N \times L N}$ is a linear operator which linearly and positively combines the spectral bands with weights equal to the samples of the sensitivity spectral pattern of the panchromatic image. The noise of the measured panchromatic image is denoted $n_{p} \sim \mathcal{N}\left(0, \sigma_{p}^{2}\right)$.

Analogously to [2], we will exploit the fact that the different spectral bands of hyperspectral images approximately share the same level lines. Such a knowledge can be integrated by comparing the gradient of the panchromatic data with the gradient of each hyperspectral image channel. A simple way to measure the discrepancy between the normal fields consist of using the function $f$ below

$$
f(u)=\sum_{l=1}^{L} \sum_{i=1}^{N}\left|\left\langle\nabla u_{l}(i), \frac{\nabla^{\perp} p(i)}{\|\nabla p(i)\|_{2}}\right\rangle_{\mathbb{R}^{2}}\right|
$$

where $\nabla=\left[\partial_{h}^{\top}, \partial_{v}^{\top}\right]^{\top}: \mathbb{R}^{N} \rightarrow \mathbb{R}^{N} \times \mathbb{R}^{N}$ is the standard discrete gradient operator, $\partial_{h}$ and $\partial_{v}$ are the horizontal and vertical gradient operators respectively, $\langle\cdot, \cdot\rangle_{\mathbb{R}^{2}}$ is the standard Euclidian dot product in $\mathbb{R}^{2}$ and $\|\cdot\|_{2}$ the associated $L_{2}$ norm. The operator $\nabla^{\perp}=\left[-\partial_{v}^{\top}, \partial_{h}^{\top}\right]^{\top}: \mathbb{R}^{N} \rightarrow \mathbb{R}^{N} \times \mathbb{R}^{N}$ returns for each pixel a vector orthogonal to the gradient. Functional $f$ has many attractive properties: it is convex in $u$ and it can be shown to have a meaning in the continuous setting for bounded variation functions.

In natural scenes, the gradient can be very low in image areas corresponding to homogeneous radiometry of the scene. In such a case, $f$ does not provide much information and an additional regularizing term should be added in the variational formulation. In this work, we use a standard total variation regularizer [5], commonly used for such purposes and adapted in Eq. 4 to multiband images

$$
T V(u)=\sum_{l=1}^{L} \sum_{i=1}^{N}\left\|\left(\nabla u_{l}\right)(i)\right\|_{2}
$$

where $\|\cdot\|_{2}$ is $L_{2}$-norm in $\mathbb{R}^{2}$. The proposed variational formulation for the hyperspectral pan-sharpening problem is as follows:

$$
\begin{aligned}
\hat{u}= & \underset{u}{\operatorname{argmin}} \gamma f(u)+(1-\gamma) T V(u) \\
\text { s.t. } & \left\|x_{l}-\mathbf{D H}_{s} u_{l}\right\|_{2}^{2} \leq M \sigma_{x_{l}}^{2}, \forall l \in[1, \ldots, L] \\
& \|p-\mathbf{G} u\|_{2}^{2} \leq N \sigma_{p}^{2}
\end{aligned}
$$

where $\|\cdot\|_{2}$ denotes the $L_{2}$ norm in $\mathbb{R}^{M}$ or $\mathbb{R}^{N}$. In this formulation, $\gamma \in[0,1]$ fixes a balance between the two terms $f$ and $T V$. The fit-to-data terms are constraints deriving from the physical models (Eq. 1 and 2). The parameters $\left\{\sigma_{x_{l}}\right\}_{l \in\{1, \ldots, L\}}$ and $\sigma_{p}$ can be a priori given or estimated, which is a strong asset of the variational constrained formulation.

\section{ADMM BASED OPTIMIZATION}

The proposed algorithm is called TVLCSP (for Total Variation iso-gray Level-set Curves Spectral Pattern) and its pseudocode is given in the procedure TVLCSP. A variant (called TVLC) not considering the sensitivity spectral and fit-topanchromatic data has been developed but is not presented here.

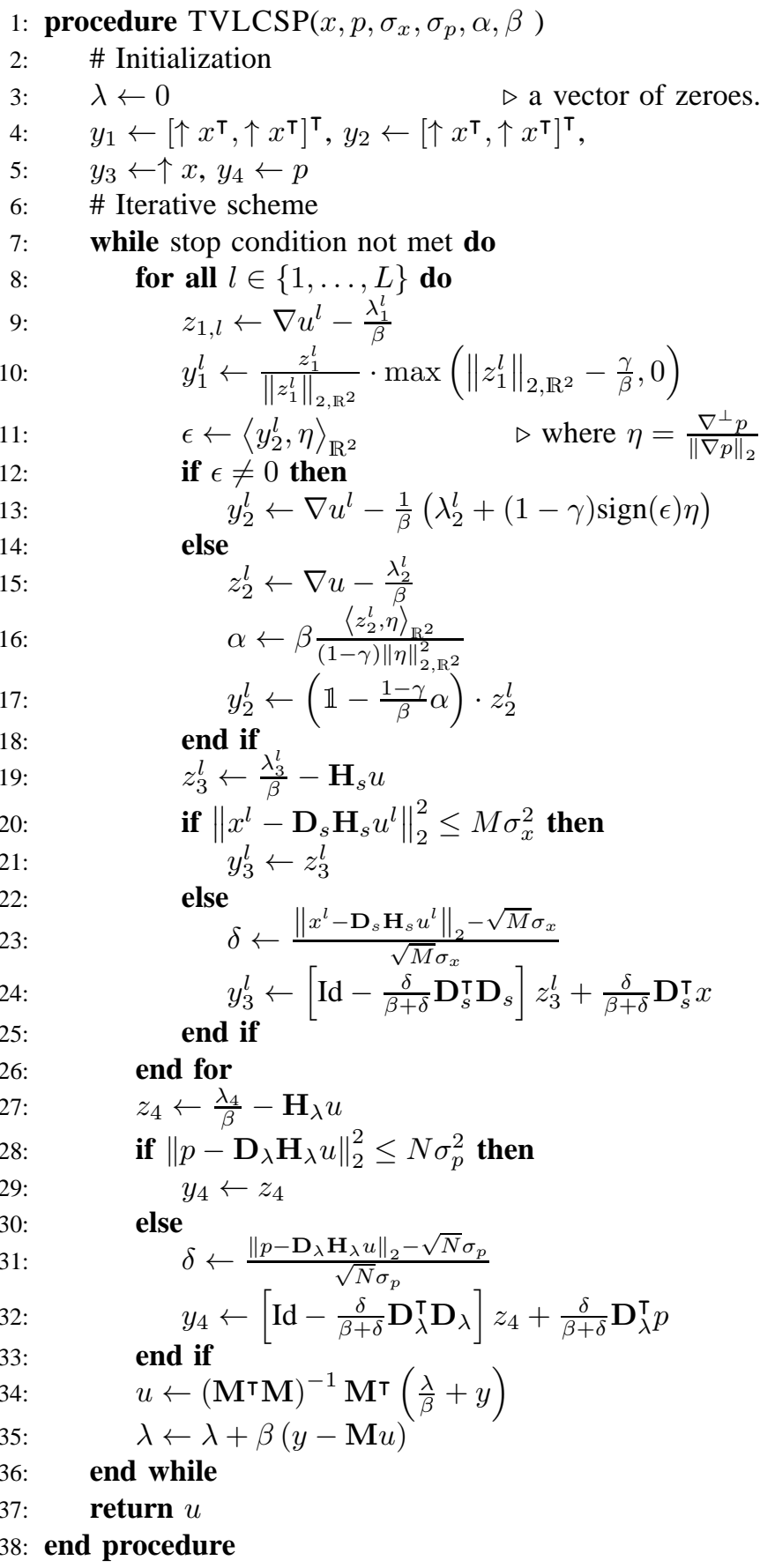

In the procedure, the vectors $\mathbb{1}$ and matrices Id are vectors of ones and identity matrices. Their dimensions depend on the context. $\forall k \in\{1,2,3\}, y_{k}=\left[y_{k, 1}^{\top}, \ldots, y_{k, L}^{\top}\right]^{\top}$ and $\lambda_{k}=\left[\lambda_{k, 1}^{\top}, \ldots, \lambda_{k, L}^{\top}\right]^{\top}$ such that $\forall l \in\{1, \ldots, L\}, \forall k \in$ $\{1,2\}, y_{k, l}, \lambda_{k, l} \in \mathbb{R}^{N} \times \mathbb{R}^{N}$ and $y_{3, l} \in \mathbb{R}^{N}$. We define $\lambda=$ $\left[\lambda_{1}^{\top}, \ldots, \lambda_{4}^{\top}\right]^{\top}$. We can note that the operator $\mathbf{G}: \mathbb{R}^{L N} \rightarrow \mathbb{R}^{N}$ can be decomposed as:

$$
\mathbf{G}=\mathbf{D}_{\lambda} \mathbf{H}_{\lambda}
$$

where $\mathbf{H}_{\lambda}: \mathbb{R}^{L N} \rightarrow \mathbb{R}^{L N}$ is a circulant matrix associated with a spatially invariant convolution kernel defined by the sensitivity spectral pattern of the panchromatic image and $\mathbf{D}_{\lambda}$ : 


\begin{tabular}{|c|c|c|c|c|c|}
\hline $\mathrm{q}$ & $\beta$ & $\gamma$ & $\sigma_{p}$ & $\sigma_{\tilde{\mathbf{u}}}$ & \#iter \\
\hline 4 & 1000 & 0.01 & 0.0001 & 0.0001 & $300 \& 3000$ \\
\hline
\end{tabular}

TABLE I

PARAMETERS OF TVLCSP FOR THE TESTS ON THE CUPRITE SCENE.

$\mathbb{R}^{L N} \rightarrow \mathbb{R}^{N}$ is a spectral decimation operator.

Finally, we define the matrix

$\mathbf{M}=\left[\left(\nabla \pi_{1}\right)^{\top}, \ldots,\left(\nabla \pi_{L}\right)^{\top},\left(\nabla \pi_{1}\right)^{\top}, \ldots,\left(\nabla \pi_{L}\right)^{\top}, \mathbf{H}_{s}^{\top}, \mathbf{H}_{\lambda}^{\top}\right]^{\top}$

The up-sampling operator $\uparrow: \mathbb{R}^{L M} \rightarrow \mathbb{R}^{L N}$ spatially upsamples a vectorized hyperspectral image by a factor $q$ and the vector $\mathbb{1}$ is a vector of ones of suitable dimension. The multiplications and divisions are element-wise. The ADMM procedure introduces an internal parameter, denoted $\beta$, whose value impacts the convergence speed.

Note that the update rule of $u$ (line 34 in the procedure) can be computed in the Fourier domain. More precisely, since $\mathbf{M}^{\top}$ and $\mathbf{M}^{\top} \mathbf{M}$ are circulant matrices (as concatenation and summation of circulant matrices, respectively), the left product by $\mathbf{M}^{\top}$, the inversion of $\mathbf{M}^{\top} \mathbf{M}$ and the left product by $\left(\mathbf{M}^{\top} \mathbf{M}\right)^{-1}$ can be performed in the Fourier domain.

matrix $\mathbf{M}$ is a concatenation of circulant matrices (Eq. 6), each associated with a convolution-type operation either in the two spatial dimensions (case of all matrices in Eq. 6 but $\mathbf{H}_{\lambda}^{\boldsymbol{\top}}$ ) or in the spectral dimension (case of $\mathbf{H}_{\lambda}^{\top}$ ). Thus, the left side product by $\mathbf{M}^{\top}$ is in the Fourier domain. Additionally, $\mathbf{M}^{\top} \mathbf{M}$ is a summation of circulant matrices, which is also circulant so the left-product by its inverse can be computed in the Fourier domain too. Thus, line 34 of the procedure should be replaced by:

$$
u=\mathcal{F}^{-1}\left(\frac{\mathcal{F}\left(\mathbf{M}^{\top}\right) \cdot \mathcal{F}\left(\frac{\lambda}{\beta}+y\right)}{\mathcal{F}\left(\mathbf{M}^{\top} \mathbf{M}\right)}\right)
$$

where $\mathcal{F}$ and $\mathcal{F}^{-1}$ represent the Fourier transform and its inverse, respectively.

Currently, the stop condition is a number of iterations but another approach could be based on the stationary of $y, \lambda$ and $u$.

\section{EXPERIMENTAL RESULTS}

We present here results of TVLCSP on AVIRIS [6] and simulated HypXim [7] data. We have first extracted a selection of the Cuprite scene (AVIRIS) which represents a mineral area. The 224-spectral band data has been preprocessed and simulated as follows. 1 - Absorption spectral bands have been removed (bands: $1-6,106-114,152-170$ and $215-224)$ to get a reference high resolution hyperspectral image $u_{\text {ref. }} 2$ - A convex combination of the spectral bands of $u_{\text {ref }}$ gives the simulated panchromatic data $p$. The weights are the coefficients of the vector $\mathrm{g}=\left[\frac{1}{80} \ldots \frac{1}{80}\right] .3$ - The low resolution hyperspectral image $x$ has been obtained from Eq. 1 without noise and with $\mathbf{H}_{s}$ representing an average filter.

The chosen algorithm parameters are given in Table [] Visual results are presented in Fig. 1.

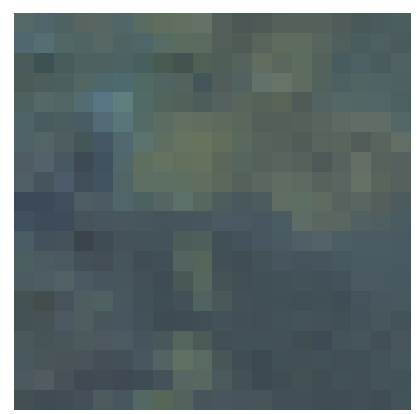

(a) HX LR

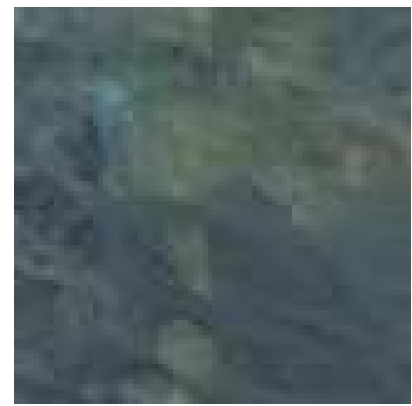

(c) Wavelet HR estimation

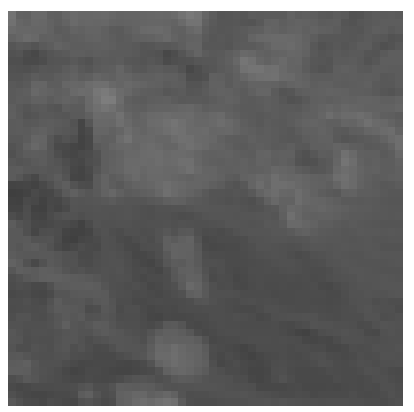

(b) Panchromatic HR

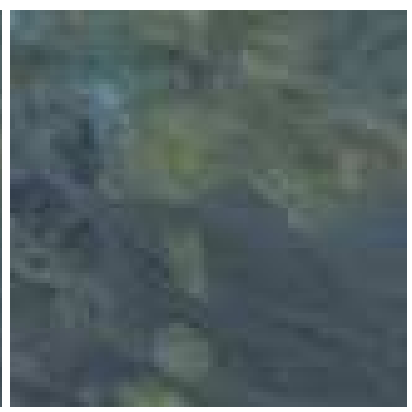

(d) TVLCSP HR estimation
Fig. 1. Cuprite scene and processing with wavelet and TVLCSP, for a resolution ratio $q=4$

\begin{tabular}{|l|c|c|c|}
\cline { 2 - 3 } \multicolumn{1}{c|}{} & \multicolumn{2}{c|}{ TVLCSP } & \multirow{2}{*}{ Wavelet } \\
\cline { 2 - 3 } \multicolumn{1}{c|}{} & $\# 300$ & $\# 3000$ & \\
\hline RMSE $(\times 100)$ & $\mathbf{0 . 4 8}$ & 0.59 & 0.91 \\
ERGAS & $\mathbf{5 . 4 5}$ & 6.68 & 10.3 \\
SAM & $\mathbf{0 . 6 1}$ & 0.70 & 0.88 \\
FCC $(\times 100)$ & $\mathbf{9 9 . 3}$ & 99.0 & 99.1 \\
$D_{s}(\times 100)$ & 1.15 & $\mathbf{0 . 8 9}$ & 2.35 \\
$D_{\lambda}(\times 100)$ & 1.82 & $\mathbf{1 . 2 2}$ & 4.43 \\
\hline
\end{tabular}

TABLE II

PERFORMANCES OF PAN-SHARPENING ALGORITHMS ON THE CUPRITE SUBIMAGE.

In Table П we present quantitative evaluation and comparison with a wavelet-based pan-sharpening method [1] using usual performance metrics: 1 - global quality metrics RMSE and ERGAS, 2 - spectral quality metrics SAM and the spectral dispersion the spatial dispersion $D_{\lambda}[\overline{8}]$ ), and 3 - spatial quality metrics FCC [9] and spatial dispersion $D_{s}$ [8]. Note that $D_{\lambda}$ and $D_{s}$ are metrics without reference (ground truth high resolution hyperspectral image) requirement, which is relevant where no reference is available or when the reference is likely to introduce error in comparison (case of our HypXim data) due to noise.

Additionally, TVLCSP has been tested on simulated HypXim data. They have been simulated from data acquired in the framework of the Pléiades program. The scene is located in Namibia and a sub-scene has been extracted. Some characteristics of the considered data are given in Table III] The considered sensitivity spectral pattern is shown in Fig. 2 a). We see that only some (20) of the spectral bands contribute to the panchromatic data thus only 20 non-zero coefficients in $\mathrm{g}$ and the presented results only concerns these bands. Note that the hyperspectral sensor spatial Point Spread Functions (PSF) has been supposed Gaussian spectrally and spatially invariant, 


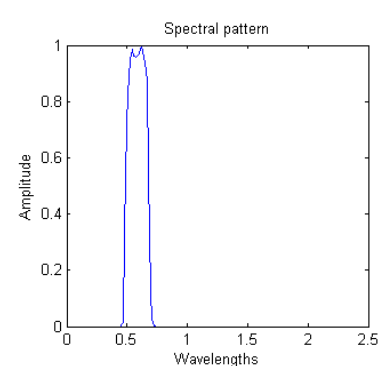

(a) Panchromatic spectral pattern

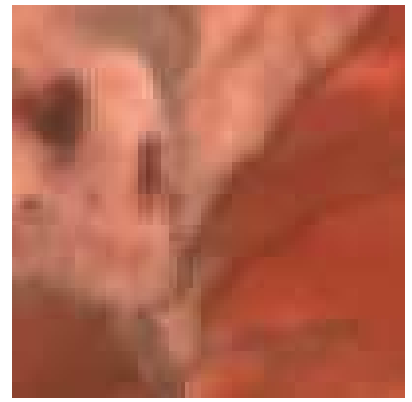

(c) LR HSI - q=2

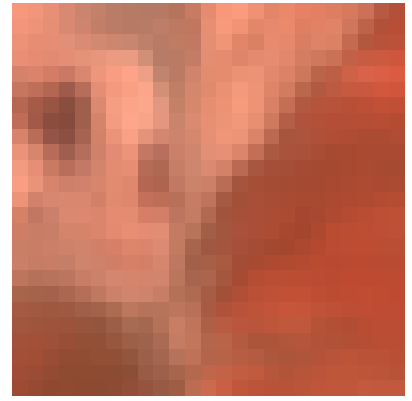

(e) LR HSI - q=4

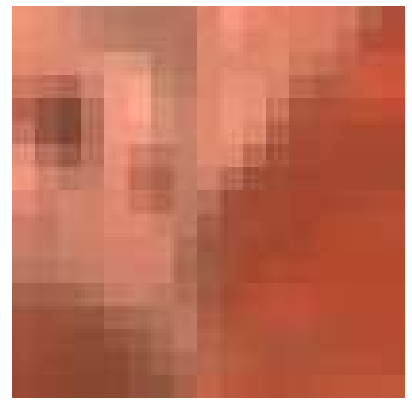

(g) LR HSI - q=6

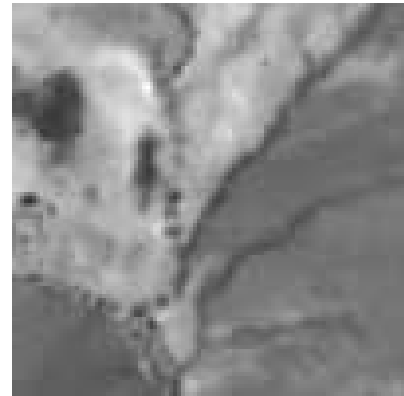

(b) Panchro

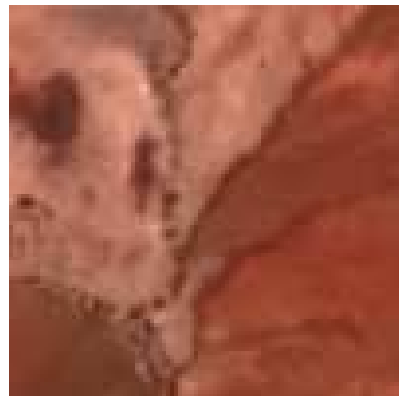

(d) TVLCSP - q=2

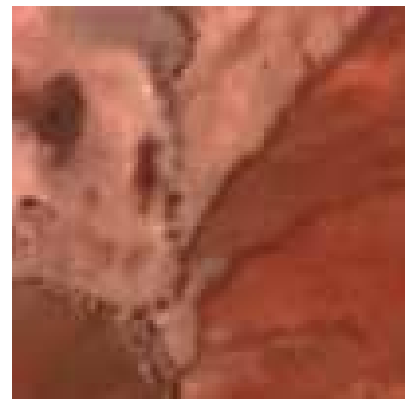

(f) TVLCSP - q=4

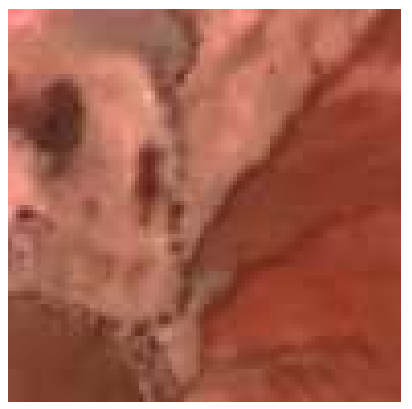

(h) TVLCSP - q=6

Fig. 2. HypXim scene and processing with TVLCSP, for resolution ratios $q=2$ and $q=6$.

\begin{tabular}{|c|c|c|}
\hline$q$ & $\begin{array}{c}\text { Spatial } \\
\text { resolution }(\mathrm{m})\end{array}$ & Sumulated sensor \\
\hline 1 & 4.80 & Panchromatic sensor \\
1 & 4.80 & Reference \\
2 & 9.60 & HypXim P (Performance concept) \\
4 & 19.20 & HypXim C (Challenging concept) \\
6 & 28.80 & ENMAP \\
\hline
\end{tabular}

TABLE III

CHARACTERISTICS OF THE SIMULATED HYPXIM AND PANCHROMATIC DATA.

\begin{tabular}{|l|c|c|c|}
\cline { 2 - 4 } \multicolumn{1}{c|}{} & $q=2$ & $q=4$ & $q=6$ \\
\hline RMSE $\times 100$ & 1.73 & 2.08 & 2.29 \\
ERGAS & 11.5 & 28.5 & 47.0 \\
SAM & 1.57 & 1.50 & 1.64 \\
FCC $\times 100$ & 97.6 & 97.3 & 97.2 \\
$D_{s} \times 100$ & 3.05 & 4.28 & 4.59 \\
$D_{\lambda} \times 100$ & 3.41 & 2.30 & 1.82 \\
\hline
\end{tabular}

TABLE IV

PERFORMANCES OF TVLCSP ON THE HyPXIM SUB-IMAGE

with a parameter tuned experimentally. The visual results are presented in Fig. 2 and the corresponding performance metrics are given in Table IV We see that the method works well for many resolution rations. However, the results on HypXim are not as good as those on AVIRIS, probably due to our approximation hypotheses on the sensor parameters and to the presence of noise. Note that the simulated reference image is corrupted by sensor noise whereas TVLCSP provides relatively denoised data estimations, which introduces lack of confidence in the performance metrics values.

\section{CONCLUSION}

We have tackled the pan-sharpening problem using a variational convex constrained approach with an objective based on the conservation of the set of iso-gray-level lines among spectral bands and total variation. The fit-to-data constraints have been mathematically considered as such and are based on the signal model and the sensor parameters, including noise statistics. An ADMM scheme has been developped, called TVLCSP and evaluated on AVIRIS and HypXim simulated data.

\section{ACKNOWLEDGMENT}

To cite this work, please use the reference [10]. The authors would like to thank the CNES for initializing and funding the study and providing HypXim simulated data.

\section{REFERENCES}

[1] T. Ranchin and L. Wald, "Fusion of high spatial and spectral resolution images: the arsis concept and its implementation," PERRS, vol. 66, no. 1, pp. 49-61, 2000.

[2] C. Ballester, V. Caselles, , L. Igual, and J. Verdera, "A variational model for p+xs image fusion," IJCV, vol. 69, no. 1, p. 4358, 2006.

[3] M. Joshi and A. Jalobeanu, "MAP estimation for multiresolution fusion in remotely sensed images using an IGMRF prior model," IEEE TGRS, vol. 48, no. 3, pp. 1245-1255, 2010.

[4] V. Caselles, B. Coll, and J.-M. Morel, "Geometry and color in natural images," JMIV, vol. 16, no. 2, pp. 89-105, 2002.

[5] L. Rudin, S. Osher, and E. Fatemi, "Nonlinear total variation based noise removal algorithms," Physica D, vol. 60, no. 1-4, pp. 259-268, 1992.

[6] "Aviris," http://aviris.jpl.nasa.gov/.

[7] R. Marion, V. Carrere, S. Jacquemoud, S. Chevrel, P. Prastault, M. D'oria, P. Gilouppe, S. Hosford, B. Lubac, and A. Bourguignon, "Hypxim: A new hyperspectral sensor combining science/defence applications," in WHISPERS, 2011.

[8] L. Alparone, B. Aiazzi, S. Baronti, A. Garzelli, F. Nencini, and M. Selva, "Multispectral and panchromatic data fusion assessment without reference," PERRS, vol. 74, no. 2, pp. 193-200, 2008.

[9] Z. Zhou, D. Civico, and J. Silander, "A wavelet transform method to merge landsat TM and SPOT panchromatic data," IJRS, vol. 19, no. 4, 1998.

[10] A. Huck, F. de Vieilleville, P. Weiss, and M. Grizonnet, "Hyperspectral pan-sharpening: a variational convex constrained formulation to impose parallel level lines, solved with ADMM," in WHISPERS, 2014. 